\title{
Deterministic Length Reduction: Fast Convolution in Sparse Data and Applications
}

\author{
Amihood Amir* \\ Oren $\operatorname{Kapah}^{\dagger}$ \\ Ely Porat \\ Bar-Ilan University \\ Bar-Ilan University \\ Bar-Ilan University \\ and \\ Georgia Tech
}

\begin{abstract}
In this paper a deterministic algorithm for the length reduction problem is presented. This algorithm enables a new tool for performing fast convolution in sparse data. While the regular fast convolution of vectors $V_{1}, V_{2}$ whose sizes are $N_{1}, N_{2}$ respectively, takes $O\left(N_{1} \log N_{2}\right)$ using FFT, the proposed algorithm performs the convolution in $O\left(n_{1} \log ^{3} n_{1}\right)$, where $n_{1}$ is the number of non-zero values in $V_{1}$. This algorithm assumes that $V_{1}$ is given in advance, and the $V_{2}$ is given in running time. This running time is achieved using a preprocessing phase on $V_{1}$, which takes $O\left(n_{1}^{2}\right)$ if $N_{1}$ is polynomial in $n_{1}$, and $O\left(n_{1}^{4}\right)$ if $N_{1}$ is exponential in $n_{1}$ (which is rarely the case in practical applications).

This tool is used to obtain faster results for several well known problems, such as the $d$ Dimensional Point Set Matching and Searching in Music Archives.
\end{abstract}

\section{Introduction}

The $d$-Dimensional point set matching problem, and its generalization - the $d$-Dimensional sparse wildcard matching problem, serve as powerful tools in numerous application domains. In the dDimensional point set matching problem, two sets of points $T, P \in \mathbb{N}^{d}$ consisting of $n, m$ points, respectively, are given. The goal is to determine if there is a rigid transformation under which all the points in $P$ are covered with points in $T$.

The d-Dimensional sparse wildcard matching problem is similar, except that every point in $\mathbb{N}^{d}$ is associated with a value. A match is declared if the values of coinciding points are equal.

Among the important application domains to which these problems contribute are the following:

\footnotetext{
*Department of Computer Science, Bar-Ilan University, Ramat-Gan 52900, Israel, +972 3 531-8770; amir@cs.biu.ac.il; and College of Computing, Georgia Tech, Atlanta, GA 30332-0280. Partly supported by NSF grant CCR-01-04494 and ISF grant 35/05.

${ }^{\dagger}$ Dept. of Computer Science, Bar-Ilan U., 52900 Ramat-Gan, Israel, (972-3)531-8408, kapaho@cs.biu.ac.il.

${ }^{\ddagger}$ Department of Computer Science, Bar-Ilan University, 52900 Ramat-Gan, Israel, (972-3)531-7620; porately@cs.biu.ac.il.
} 


\section{Model based object recognition:}

In model-based recognition problems, a model of an object undergoes some geometric transformation that maps the model into a sensor coordinate system. This may be an image plane, a coordinate system from a 3d scanner, or a multidimensional feature space. When seeking occurrences of an object in a larger space, one needs to solve a problem similar to the point-set matching problem. For many applications one needs affine transformations, rather than just translations, in order to declare a match (e.g. $[8,3])$. Nevertheless, the point-set matching problem is a natural tool to use when seeking occurrences of an object in feature space, or in applications where translation alone suffices [9].

\section{Image Registration:}

The task of automated image registration can be divided into two major components: (1) the extraction of features from images; and (2) the search among the extracted features for the matching pairs that represent a sought object in the space of the images to be matched [2]. Clearly, one may use point-set matching to this end.

\section{Pharmacophore Identification:}

One of the purposes of structural computational biology is the development of pharmaceutical drugs. A pharmacophore is a three dimensional map of biological properties common to all active conformations of a set of ligands which exhibit a particular activity. Conceptually, a pharmacophore is a distillation of the functional attributes of ligands which accomplish a specific task. Pharmacophores are conceptual templates for drug design. Once extracted, a pharmacophore can be used as a model for the design of other molecules that can accomplish the same activity. The pharmacophore is an abstraction of a molecule, which must be congruent to the functional components of another desired molecule [6]. Again, the point-set matching is a tool towards solution.

\section{Searching In Music Archives}

Currently, many music archives are stored in MIDI format. In this representation, among other information, the pitch level of every played note is stored with a time-stamp. This information can be represented as points in a 2-Dimensional space where the axes are time and pitch. The problem of seeking a given melody in the archive is immediately reduced to a point set matching problem in 2-dimensional space [10].

Given the importance of the point-set matching problem it is not surprising that the problem has been widely considered in the literature in many variations, not the least of which in the algorithms literature. In [9] Cardoze and Schulman used a randomized algorithm to reduce the space size of $T, P$ and then apply solve the problem in the reduced space. In [4] Cole and Hariharan proposed a solution to the d-Dimensional Sparse Wildcard Matching. Their solution consists of two steps. The first step is a Dimension Reduction where the inputs $T, P$ are linearized into row vectors $T^{\prime}, P^{\prime}$ of size polynomial in the number of non-zero values. The second step was a Length Reduction where each of the row vectors $T^{\prime}, P^{\prime}$ was replaced by $\log n$ short vectors of size $O(n)$ where $n$ is the number of non-zeros. The idea is that the mapping to the short vectors preserves the distances in the original vectors, thus the problem is reduced to a matching problem of short vectors, to which efficient solutions exist. The problem with the length reduction idea is that more then one point can be mapped into the same location, thus it is no longer clear whether there is indeed a match in 
the original vectors. The proposed solution of Cole and Hariharan was to create a set of $\log n$ pairs of vectors using $\log n$ hash function rather then a single pair of vectors. Their scheme reduced the failure probability. To our knowledge, all currently known efficient solutions to the problem are randomized.

In this paper we present the first deterministic algorithm for finding $\log n$ hash functions that reduce the size of the vectors to $O(n \log n)$. We guarantee that each non-zero value appears with no collisions in at least one of the vectors, thus eliminating the possibility of en error. Our algorithm also has the advantage that it is surprisingly simple and easy to implement.

In Section 4 we use our length reduction algorithm to solve the Sparse Convolution problem posed in [7], where the aim is to find the convolution vector $W$ of the two vectors $V_{1}, V_{2}$. It is assumed that the two vectors are not given explicitly, rather they are given as a set of (index, value) pairs. Using the Fast Fourier Transform (FFT) algorithm, the convolution can be calculated in running time $O\left(N_{1} \log N_{2}\right)$ [5]. In our context, though, the vectors $V_{1}, V_{2}$ are sparse. The aim of the algorithm is to compute $W$ in time proportional to the number of non-zero entries in $W$, which may be significantly smaller than $O\left(N_{1}\right)$.

It should be noted that our algorithm assumes that the point-set matching is done on an archive, i.e. we assume a given set of points which we pre-process in time quadratic in the number of non-zero points. Subsequently, we can solve any point-set matching query in time linear times polylog of the number of points. In the sparse convolution setting this means that the larger vector is fixed. The best known current deterministic solution takes time proportional to the product of the number of non-zero values in the two vectors, even if the same vector is re-used.

In Section 5 we demonstrate our fast sparse convolution algorithm on two applications: the $d$ Dimensional Point Set Matching and Searching in Music Archives problems. In both cases there is no known deterministic algorithm whose time is better than $O\left(n_{1} n_{2}\right)$. Our algorithm offers a solution in time $O\left(n_{1} \log ^{3} n_{1}\right)$.

\section{Preliminaries and Notations}

Throughout this paper, a capital letter (usually $N$ ) is used to denote the size of the vector, which is equivalent to the largest index of a non-zero value, and a small letter (usually $n$ ) is used to denote the number of non-zero values. It is assumed that the vectors are not given explicitly, rather they are given as a set of (index, value) pairs, for all the non-zero values.

A convolution uses two initial functions, $v_{1}$ and $v_{2}$, to produce a third function $w$. We formally define a discrete convolution.

Definition 1 Let $V_{1}$ be a function whose domain is $\left\{0, \ldots, N_{1}-1\right\}$ and $V_{2}$ a function whose domain is $\left\{0, \ldots, N_{2}-1\right\}$. We may view $V_{1}$ and $V_{2}$ as arrays of numbers, whose lengths are $N_{1}$ and $N_{2}$, respectively. The discrete convolution of $V_{1}$ and $V_{2}$ is the polynomial multiplication

$$
W[j]=\sum_{i=0}^{N_{2}-1} V_{1}[j+i] V_{2}[i] .
$$


In the general case, the convolution can be computed by using the Fast Fourier Transform (FFT) [5]. This can be done in time $O\left(N_{1} \log N_{2}\right)$, in a computational model with word size $O\left(\log N_{2}\right)$. In the sparse case, many values of $V_{1}$ and $V_{2}$ are 0 . Thus, they do not contribute to the convolution value. In our convention, the number of non-zero values of $V_{1}\left(V_{2}\right)$ is $n_{1}\left(n_{2}\right)$. Clearly, we can compute the convolution in time $O\left(n_{1} n_{2}\right)$. The question posed by Muthukrishnan [7] is whether the convolution can be computed in time $o\left(n_{1} n_{2}\right)$.

Cole and Hariharan's suggestion was to use length reduction. Suppose we can map all the nonzero values into a smaller vector, say of size $O\left(n_{1} \log n_{1}\right)$. Suppose also that this mapping is alignment preserving in the sense that applying the same transformation on $V_{2}$ will guarantee that the alignments are preserved. Then we can simply map the the vectors $V_{1}$ and $V_{2}$ into the smaller vectors and then use FFT for the convolutions on the smaller vectors, achieving time $O\left(n_{1} \log ^{2} n_{1}\right)$.

The problem is that to-date there is no known mapping with that alignment preserving property. Cole and Hariharan [4] suggested a randomized idea that answers the problem with high probability. The reason their algorithm is not deterministic is the following: In their length reduction phase, several indices of non-zero values in the original vector may be mapped into the same index in the reduced size vector. If the index of only one non-zero value is mapped into an index in the reduced size vector, then this index is denoted as singleton and the non-zero value is said to appear as a singleton. If more then one non-zero value is mapped into the same index in the reduced size vector, then this index is denoted as multiple. The multiple case is problematic since we can not be sure of the right alignment. Fortunately, Cole and Hariharan showed a method whereby in $O\left(\log n_{1}\right)$ tries, the probability that some index will always be in a multiple situation is small. In the next section we present a deterministic solution to the multiple problem.

\section{Deterministic Length Reduction}

Let $P_{i}$ be a prime number. Construct the array $V_{i}$ achieved by mapping the (index, value) pair $(i, v)$ to $\left(i \bmod P_{i}, v\right)$. Call array $V_{i}$ the reduced length vector by $P_{i}$.

Our idea is to deterministically find a set $\left\{P_{1}, \ldots, P_{i}, \ldots, P_{\log n_{1}}\right\}$ of prime numbers in the preprocessing phase, such that when constructing the reduced length vectors $V_{1, i}, i=1, \ldots, \log n_{1}$, each non-zero input is guaranteed to appear as a singleton in at least one of the new vectors. Note that in order to save space, there is no need to keep the new vectors, since they can be reconstructed in running time given the set of prime numbers.

The rest of this section is devoted to finding this set of prime numbers. We distinguish between two cases.

1. The size of the original vector is polynomial in $n_{1}\left(N_{1}<n_{1}^{c}\right)$.

2. The size of the original vector is exponential in $n_{1}$.

Most of the practical applications fall into the first category. In addition, it is important to note that in case of $N_{1}$ exponential in $n_{1}$, just encoding the pairs requires $O\left(n_{1}^{2}\right)$ space. However, we provide an algorithm for the second case for the sake of completeness. This algorithm works in 
the arithmetic model, i.e. we assume that an address can fit in a computer word and that a word operation can be done in constant time.

\subsection{Case 1: $N_{1}$ is polynomial in $n_{1}$}

In the first case we find a set of $\log n_{1}$ prime numbers which enables the creation of $\log n_{1}$ vectors of size $O\left(n_{1} \log n_{1}\right)$ such that each non-zero appears in at least one of the vectors as a singleton. For every prime number $P_{i}$ the corresponding vector $V_{1, i}$ is created by setting the index of each non-zero value $(i, v)$ to be the $i \bmod P_{i}$.

Observation 1 For every $i$, two non-zeros can be mapped into the same location in $V_{1, i}$ only if $P_{i}$ divides the distance between them.

The following lemma is crucial to our algorithm.

Lemma 1 Any two non-zeros can be mapped to the same location in at most c vectors.

Proof: The distance between any two non-zeros is bounded by the size of the original vector $N_{1}<n_{1}^{c}$, thus the number of different prime numbers greater then $n_{1}$ which divide the distance between them is bounded by $\log _{n_{1}} n_{1}^{c}=c$.

Since any non-zero can be mapped into the same location with at most $n_{1}-1$ other non-zeros, and with each of them at most $c$ times, due to Lemma 1, then we get the following Corollary:

Corollary 1 Any non-zero can appear as a multiple in not more then $n_{1} c$ vectors.

In the preprocessing step $2 c n_{1}$ prime numbers of size $O\left(n_{1} \log n_{1}\right)$ are selected. Corollary 1 assures us that they can be selected in any way. For example, the first $2 c n_{1}$ prime numbers which are greater than $n_{1} \log n_{1}$ can be chosen. For each prime number $P_{i}$ the reduced length vector $V_{i}$ is constructed. Then a set of $\log n_{1}$ prime numbers is chosen from them such that each of the non-zeros will appear as a singleton in at least one of the corresponding reduced length vectors.

The selection of the $\log n_{1}$ prime number is done as follows: Construct table $A$ with $n_{1}$ columns and $2 c n_{1}$ rows. Row $i$ correspond to a prime number $P_{i}$ and its reduced length vector $V_{1, i}$. A column corresponds to a non-zero value in $V_{1}$. The value of $A_{i j}$ is set to 1 if non-zero $j$ appears as a singleton in vector $V_{1, i}$. Due to Corollary 1 , the number of zeros in each column can not exceed $n_{1} c$. Thus, in each column there are 1's in at least half of the rows, which means that the table is at least half full. Since the table is at least half full there exist a row in which there is one in at least half of the columns. The prime number which generated this row is chosen. All the columns where there was a 1 in the selected row are deleted from the table.

Recursively another prime number is chosen and the table size is halved again, until all the columns are deleted. Since at each step at least half of the columns are deleted, the number of prime number chosen can not exceed $\log n_{1}$.

The algorithm appears in detail below. 
Algorithm $-N_{1}<n_{1}^{c}$, for constant $c$

1. Create a matrix $A$ of $n_{1}$ columns and $2 c n_{1}$ rows.

2. For $i=1$ to $2 c n_{1}$

(a) Select a new prime number $P_{i}$ of size $O\left(n_{1} \log n_{1}\right)$.

(b) Create a new vector $V_{1, i}$ from the input by mapping the index $\ell$ of each non-zero pair $(\ell, v)$ to: $\ell \bmod P_{i}$.

(c) For each non-zero input $j$ from 1 to $n_{1}$

(d) If $j$ appears as a singleton in $V_{1, i}$ set $A_{i j}=1$, else set $A_{i j}=0$

3. Choose a row which is at least half full.

4. Delete all the columns in which 1 appears in the selected row from the matrix.

5. while the matrix is not empty go to step 3 .

end Algorithm

Correctness: Immediately follows from the discussion.

Time: Creating vector $V_{1, i}$ (row $i$ ) takes $O\left(n_{1}\right)$ time. Since we start with a full matrix of $O\left(n_{1}\right)$ rows then the initialization takes $O\left(n_{1}^{2}\right)$ time. Choosing the $\log n_{1}$ primes is done recursively. The recurrence is:

$$
t\left(n_{1}^{2}\right)=n_{1}^{2}+t\left(\frac{n_{1}^{2}}{2}\right)
$$

The closed form of this recurrence is $O\left(n_{1}^{2}\right)$.

Primality Testing: The set of prime numbers $\left\{P_{1}, \ldots, P_{\log n_{1}}\right\}$ is independent of the vector $V_{1}$. The same set can be used for all vectors with $n_{1}$ non-zero values. Thus we may assume that a prime numbers table is available. In any event, primality testing of number $n$ can be currently done in $O\left(\log ^{4+\epsilon} n\right)$ time [1], thus one can also generate prime numbers online.

\subsection{Case 2: $N_{1}$ is exponential in $n_{1}$}

In this case, the algorithm reduces the original vector to a single vector of size $O\left(n_{1}^{4}\right)$, where all the non-zeros appear as singletons, and then continues as in the first case. This is achieved by choosing a prime number of size $O\left(n_{1}^{4}\right)$, such that all of the non-zeros appear as singletons in the reduced length vector. When such a prime number is found, the algorithm in Subsection 3.1 is applied on the reduced length vector.

Finding such a prime number, which generates a reduced length vector in which all the non-zeros appear as singletons, is achieved by choosing sequentially prime numbers of size $O\left(n_{1}^{4}\right)$ and testing them.

Lemma 2 There are at most $\log N_{1}$ prime number greater than $n_{1}^{4}$ that map two non-zeros of $V_{1}$ to the same location. 
Proof: The distance between any two non-zeros is bounded by the size $N_{1}$ of the original vector. Thus there are at most $\log N_{1}$ prime numbers that divide the distance between them.

Since the number of distinct distances between any pair of non-zeros is bounded by $n_{1}^{2}$, then the number of prime numbers which generate a multiple location is bounded by $n_{1}^{2} \times \log N_{1}$. Thus, at most $n_{1}^{2} \times \log N_{1}$ prime numbers need to be tested before finding the desired prime number.

After finding such a prime number $Q$, a vector $T^{\prime}$ of size $2 Q$ is created for the text, where each non-zero from index $l$ in the text is mapped into two indices: (1) $l \bmod Q \cdot(2)(l \bmod Q)+Q$.

The vector $P^{\prime}$ which is created from the pattern is of size $Q$, in which, each non-zero from index $l$ in the pattern is mapped into $l \bmod Q$.

After obtaining the vectors $T^{\prime}$ and $P^{\prime}$, the algorithm continues as described in the previous section on the newly obtained vectors.

Algorithm $-N_{1}$ is exponential in $n_{1}$

1. Select a new prime number $Q$ of size $O\left(n_{1}^{4}\right)$.

2. Create a new vector $V_{1}^{\prime}$ from the input by mapping the index $\ell$ of each non-zero pair $(\ell, v)$ to: $\ell \bmod Q$ and to: $(\ell \bmod Q)+Q$.

3. If not all the non-zeros appear as singletons in $V_{1, i}$ go to step 2 .

4. Apply the algorithm for case 1 on the resulting vector.

end Algorithm

Correctness: Immediate from the discussion.

Time: Checking if the vector contains only singletons takes time $O\left(n_{1}\right)$ thus the time spent on steps 1 and 2 is $O\left(n_{1}\right)$. we repeat these steps at most $O\left(n_{1}^{2}\right) \times \log N_{1}$ times until we find a prime number which creates a vector with no multiples, thus the total time for the algorithm is $O\left(n_{1}^{3} \times \log N_{1}\right)=O\left(n_{1}^{4}\right)$.

\section{Fast Convolution in Sparse Data Using Length Reduction}

We return to the problem of finding the convolution vector $W$ of the two vectors $V_{1}, V_{2}$. It is assumed that the two vectors are not given explicitly, rather they are given as a set of (index, value) pairs. While in the regular fast convolution the running time is $O\left(N_{1} \log N_{2}\right)$, the aim here is to compute $W$ in time proportional to the number of non-zero entries in $W$. This problem was posed in [7]. Note that the result of the convolution needs to be computed only for the locations in which every non-zero in $V_{2}$ is aligned with non-zero in $V_{1}$. In [4] Cole and Hariharan proposed a Las Vegas randomized algorithm which works in time $O\left(w \log ^{2} n_{2}\right)$ whose failure probability is inverse polynomial in $n_{2}$. We now present the first deterministic solution to this problem. 


\subsection{The Main Idea}

The algorithm works in 2 steps: (1) Find the locations in which all non-zeros from $V_{2}$ are aligned with non-zeros in $V_{1}$ [4], and (2) Calculate the desired convolution by performing convolutions over all the $\log n_{1}$ vectors $V_{1, i}, V_{2, i}$, and sum the results for every location where an alignment was found. In order not to sum the results of a non-zero value more then once, if it appears as singleton in more then one reduced size vector, its value is zeroed after the first time.

Note that when reducing the vector size, more then one index in the original vector may be mapped into the same index in the reduced size vector. It may, therefore, happen that two aligned singletons will appear in the reduced size vectors $V_{1, i}, V_{2, i}$, while in the original vectors the non-zero in $V_{2}$ was aligned with an empty index in $V_{1}$. In order to eliminate this possibility the first step is mandatory. The first step eliminates this problem due to the fact that it ensures that every non-zero in $V_{2}$ is aligned with a non-zero in $V_{1}$, thus by the property of the length reduction, every non-zero in the reduced size vector $V_{2, i}$ has to be aligned with at least the value which was aligned with it in the original vector. Thus, if there is only one non-zero in $V_{1, i}$ aligned with a non-zero in $V_{2, i}$, they must have originated from two non-zero values which where aligned in the original size vectors $V_{1}, V_{2}$.

The algorithm for the first step uses the solution of Cole and Hariharan [4] for the Shift Matching problem.

Definition 2 Let $T$ be a length $n$ text and $P$ a length $m$ pattern over alphabet $\mathbb{N} \cup\{\phi\}$, where $\phi$ is the wildcard symbol. We say that $P$ shift matches $T$ at location $i$ if there exist an integer $\ell_{i}$ such that one of the following conditions holds for all non-wildcard symbols $P[j]$ in $P$ :

1. The text character $T[i+j]$ aligned with $P[j]$ is a wildcard.

2. $T[i+j]-P[j]=\ell_{i}$.

Cole and Hariharan [4] provide an algorithm for solving this problem in time $O(n \log m)$.

Note that for a location $i$ where every non-zero $V_{2}[j]$ is aligned with a non-zero $V_{1}[i+j]$ in $V_{1}$, there exists a shift match between the indices of $V_{2}$ and the indices of $V_{1}$ and the shift is exactly $j$.

In the same paper Cole and Hariharan also give the following definition and Lemmas:

Definition $3 A$ wrap around placement of $V_{2, i}$ starting at location $\ell$ in $V_{1, i}$ is a placement such that $V_{2, i}[k]$ is aligned with $V_{1, i}\left[(\ell+k) \bmod P_{i}\right]$

Lemma 3 Consider a wrap-around placement of $V_{2, i}$ in $V_{1, i}\left[j \bmod P_{i}\right]$. Then for each $k, 0 \leq k \leq$ $N_{2}, V_{2, i}\left[k \bmod P_{i}\right]$ is aligned with $V_{1, i}\left[(j+k) \bmod P_{i}\right]$.

Lemma 4 For any location where all non-zeros from $V_{2}$ are aligned with non-zeros in $V_{1}$, if a non-zero appears as a singleton in $V_{1, i}$ then it appears as a singleton in $V_{2, i}$.

Following these lemmas, checking for existence of such an alignment is performed as follows: 
For every $P_{i}$ create the corresponding vectors $V_{1, i}, V_{2, i}$ where the indices of each non-zero are filled in the new vectors for every non-zero which appears as singleton. All the locations defined as multiples are assigned with a wildcard (zeroed). Perform a shift matching between $V_{1, i}, V_{2, i}$. Subsequently, the same vectors are created, this time with the value 1 instead of the indices, and the convolution is performed again to count the number of non-zeros which where considered in the shift match of every location.

Lemma 5 A placement of $V_{2}$ in $V_{1}$ where all non-zeros in $V_{2}$ are aligned with non-zeros in $V_{1}$ exists if and only if there exist a shift match in all of the created vectors with the same shift (the shift amount is the location where the alignment exist), and the number of non-zeros which participate is $n_{2}$.

Proof: $\Rightarrow$ Consider a location $j$ where such an alignment exists. Now consider a non-zero at location $j+k$ in $V_{1}$ which aligns with a non-zero at location $k$ in $V_{2}$. Due to the construction of the reduced vectors, there exist an $i$ such that this non-zero appears as a singleton in $V_{1, i}$, and due to Lemma 4 , the aligned non-zero, at location $k$ in $V_{2}$ appears as a singleton in $V_{2, i}$. A shift match between them will give the difference between the indices which is $j$, and this is the same for all the $n_{2}$ non-zeros which participate in this alignment.

$\Leftarrow$ Consider a shift match where the indices of $n_{2}$ distinct non-zeros in $V_{2, i}, 0 \leq i \leq \log n_{1}$ are in distance $j$ with the indices of non-zeros in $V_{1, i}$. Thus there exist $n_{2}$ non-zeros at locations $k_{1}, \ldots, k_{n_{2}}$ in $V_{2}$ and $n_{2}$ non-zeros at locations $j+k_{1}, \ldots, j+k_{n_{2}}$ in $V_{1}$. This means that at location $j$ all the non-zeros in $V_{2}$ are aligned with non-zeros in $V_{1}$.

To calculate the result of the convolution, a pair of vectors $V_{1, i}, V_{2, i}$ is created for every $P_{i}$. The values of all non-zeros are filled in the new vectors for every non-zero which appears as a singleton. All the locations defined as multiples are zeroed. Each value of non-zero in the $V_{1}$ is filled in only one of the vectors and in the rest of the vectors it is zeroed. In $V_{2}$ values are zeroed only if they appears at locations which are defined as multiples.

We are now ready to present the algorithm in detail. 


\subsection{The Algorithm}

1. For every $i=1$ to $\log n$

(a) For each non-zero value $x \in V_{1}$

i. If $V_{1, i}\left[\operatorname{index}(x) \bmod P_{i}\right]=0$ and not marked as multiple,

- Set $V_{1, i}\left[\operatorname{index}(x) \bmod P_{i}\right]=$ OriginalIndex $(x)$.

ii. Else,

- Mark it as multiple.

- Set $V_{1, i}\left[\operatorname{index}(x) \bmod P_{i}\right]=0$.

(b) For each non-zero value $x \in V_{2}$ set

i. If $V_{2, i}\left[\right.$ index $\left.(x) \bmod P_{i}\right]=0$ and not marked as multiple,

- Set $V_{2, i}\left[\operatorname{index}(x) \bmod P_{i}\right]=$ OriginalIndex $(x)$.

ii. Else,

- Mark it as multiple.

- Set $V_{2, i}\left[\operatorname{index}(x) \bmod P_{i}\right]=0$.

(c) Perform Shift-Matching.

(d) Replace all the indices in $V_{1, i}$ with the value of the non-zero and delete all the non-zeros which appeared in previous vectors.

(e) Replace all the indices in $V_{2, i}$ with the value of the non-zero and delete all the non-zeros which appeared in previous vectors.

(f) Calculate the convolution of $V_{1, i}, V_{2, i}$ using FFT.

(g) Replace all non-zeros in $V_{1, i}, V_{2, i}$ with ones.

(h) Calculate the convolution of $V_{1, i}, V_{2, i}$ using FFT.

2. Mark all locations where the results of the Shift Match is consistent over all the $\log n$ vectors.

3. For each such location

(a) Sum the results of the convolution of the ones vectors.

(b) If the sum equal $N_{2}$,

i. Sum the results of the convolution of the values vectors.

ii. Set ResultVector [shift] to be the calculated sum.

Correctness: Follows from the discussion above.

Time: We perform $O\left(\log n_{1}\right)$ convolutions of the reduced length vectors, whose lengths are $O\left(n_{1} \log n_{1}\right)$. Each convolution's time is therefore $O\left(n_{1} \log ^{2} n_{1}\right)$ for a total time of $O\left(n_{1} \log ^{3} n_{1}\right)$. 


\section{Applications}

\section{1 d-Dimensional Point Set Matching}

In this problem, the input is given as d-dimensional text and pattern $T, P \in \mathbb{N}^{d}$, of size $n, m$ respectively. The goal is to find the translations of $P$, under which each point in $P$ is covered by a point in $T$. This problem is easily solved using the algorithm for fast convolution in sparse data. The first step is a dimension reduction [4], where from the d-dimensional input, a long row vector is created. This is performed by simply concatenating the indices of each point, i.e for each point with indices $i_{1}, \ldots, i_{d}$, the index in the row vector will be $i_{1} * N^{d-1}+\ldots+i_{d} * N^{0}$. This is done for both $T, P$ creating $T^{\prime}, P^{\prime}$, where 1 is placed in each index where there is a point. Then the fast convolution as suggested in this paper is performed. Any index where the result of the convolution is $m$ gives the translation under which a match is found. The running time of this algorithm is the time of the convolution which is $O\left(n \log ^{3} n\right)$. This is the first deterministic algorithm which performs better the naive algorithm which takes time $O(n m)$.

\subsection{Searching In Music Archives}

This problem deals with archive files in MIDI format. Among other information, this format records the pitch level with the time-stamp for every note that was played. This information can be represented as points in a 2-Dimensional space where one axis is time, and the other axis of the pitch. Given such a database of melodies $D$ of $n$ notes, and a new melody $P$, the goal is to find wether $P$ appears in $D$. Usually, if the pitch was translated by a constant value, $P$ is still referred as the same melody, thus this problem is reduced to a point set matching problem in 2-dimensional space. The time of this algorithm will be $O\left(n \log ^{3} n\right)$, with a preprocessing step on the database in time $O\left(n^{2}\right)$ if $N<n^{c}$ or $O\left(n^{4}\right)$ if $N \in \operatorname{Exp}(n)$.

Another possible problem may be finding an exact match with no translation on the pitch. This can be solved by creating from the input a vector where the time is the index, and the pitch is the value. Using the fast sparse convolution algorithm presented in this paper, this problem can be solved in time $O\left(n \log ^{3} n\right)$. To our knowledge, this is the first deterministic solution to the problem whose time is $o(n m)$, where $m$ is the number of notes in the pattern melody [10].

\section{Conclusion and Open Problems}

Deterministic algorithms for Length Reduction and Sparse Convolution where presented in this paper. These can be used as tools to provide faster algorithms for several well known problems.

Several problems still remained open, such as the problem of $d$-Dimensional Point Set Matching under transformations such as rotation, or the problem of Searching in Music with Scaling (in both the pitch and the time), where nothing better then the naive algorithm is known.

Another important problem remains: Can the Length Reduction and Sparse Convolution problems be solved in real time without the need of the preprocessing step. 


\section{References}

[1] P. Berrizbeitia. Sharpening "primes is in p" for a large family of numbers. http://arxiv.org/abs/math.NT/0211334, November 2002.

[2] L. Gottesfeld Brown. A survey of image registration techniques. ACM Computing Surveys, 24(4):325$376,1992$.

[3] K.-W. Cheung, D.-Y. Yeung, and R. T. Chin. Bidirectional deformable matching with application to handwritten character extraction. IEEE Transactions on Pattern Analysis and Machine Intelligence, 24(8):1133-1139, 2002.

[4] R. Cole and R. Hariharan. Verifying candidate matches in sparse and wildcard matching. In Proc. 34st Annual Symposium on the Theory of Computing (STOC), pages 592-601, 2002.

[5] T. H. Cormen, C. E. Leiserson, and R. L. Rivest. Introduction to Algorithms. MIT Press and McGrawHill, 1992.

[6] L. Kavraki. Pharmacophore identification and the unknown receptor problem. J Comput Aided Mol Des., 16(8-9):653-681, 2002.

[7] S. Muthukrishnan. New results and open problems related to non-standard stringology. In Proc. 6th Combinatorial Pattern Matching Conference, pages 298-317. Lecture Notes in Computer Science 937, Springer-Verlag, 1995.

[8] W. J. Rucklidge. Efficient visual recognition using the hausdorff distance. Springer Verlag, 1996.

[9] L. Schulman and D. Cardoze. Pattern matching for spatial point sets. Proc. 39th IEEE FOCS, pages 156-165, 1998.

[10] E. Ukkonen, K. Lemström, and V. Mäkinen. Sweepline the music! In R. Klein, H.-W. Six, and L. Wegner, editors, Computer Science in Perspective, Essays Dedicated to Thomas Ottmann, LNCS 2598, pages 330-342. Springer-Verlag, 2003. 